\begin{tabular}{|l|l|l|l|}
\hline Eiszeitalter $u$. Gegenwart & $\mathbf{3 2}$ & $\begin{array}{c}93-108 \\
4 \mathrm{Abb} .1 \mathrm{Tab} .\end{array}$ & Hannover 1982 \\
\hline
\end{tabular}

\title{
Sedimente und Relief einer eiszeitlichen Hohlform bei Krempel (Elbe-Weser-Dreieck)
}

\author{
Udo LAde \& Horst Hagedorn *)
}

Periglacial features, pedogenesis, solifluction, paleosol, parabrown soil, podzol, soil profile, size distribution, sand, open pit mining, alluvium, depression, paleontological reconstruction, paleorelief, Warthe Glacial, Weichsel Glacial, Eem Brörup Stadial, Brörup Odderade Stadial, Holocene. North West German Lowlands, Krempel, Niedersachsen, TK 25 Nr.: 2218

Kurzfassung: Aus einer Sandgrube bei Krempel (Elbe-Weser-Dreieck) werden periglaziäre Sedimente und Reste zweier fossiler Böden des Pleistozäns, eingelagert in eine fossile Hohlform, beschrieben.

Aus der Reliefentwicklung ließ sich ein schematischer Zyklus hinsichtlich Klima, Transport- bzw. Bildungsvorgang und Sediment bzw. Bildungsprodukt ableiten:
Stadial
Hochstadial/-glazial
$\begin{array}{ll}- \text { - } & \text { Abspülung } \\ \text { - } & \text { Solifluktion } \\ - & \text { Bodenbildung }\end{array}$
$\longrightarrow$ geschichtete Sande und Kiese;
Interstadial/-glazial ——— Bodenbildung
Fließerde;
Boden.

Das Schema sollte an anderer Stelle auf seine Allgemeingültigkeit überprüft werden.

\section{[Sediments and Relief of a Glacial Hollow near Krempel (Elbe-Weser-Triangle)]}

A bstract: Periglacial sediments and relicts of two fossil soils were found in an infilled hollow in a sand-pit near Krempel (Elbe-Weser-triangle). The lower soil has been indirectly dated by means of a neighboring peat in the center of the hollow, whereas the upper soil probably developed in the Brörup interstadial.

The following idealized cycle can be derived concerning climate, transport or formational process, and sediment or formational product:
Stadial
Plenistadial/pleniglacial
- - slope wash
- - solifluction
Interstadial/interglacial $\longrightarrow$ - soil formation $\longrightarrow \longrightarrow$ soil.
$\longrightarrow$
$\longrightarrow \longrightarrow$ bedded sands and gravels;

The model should be tested for its general relevance in other places.

\section{Inhaltsverzeichnis}

1. Einleitung

2. Arbeitsmethodik

3. Lage, Geologie und Morphologie

4. Beschreibung

4.1. Sandgrube Sonnen-Berg-NW

4.2. Profile Sonnen-Berg-NW

4.3. Reliefentwicklung

5. Schriftenverzeichnis

\section{Einleitung}

Abflußlose Hohlformen sind Sedimentfallen und eignen sich daher für Untersuchungen der Abtragungs- und Ablagerungsvorgänge in der näheren Umgebung. Eine in ihrem Randbereich aufgeschlossene, heute nicht mehr existierende Hohlform fanden wir in einer

*) Anschriften der Autoren: Dr. U. L a d e u. Prof. Dr. H. H a g e do rn, Geographisches Institut der Universität, Am Hubland, D-8700 Würzburg. 
kleinen Sandgrube nordwestlich des Sonnen-Berges bei Krempel. Die Grube bekam die Bezeichnung "Sonnen-Berg-NW“ und enthielt neben periglaziären Sedimenten Reste zweier fossiler Böden. Im zentralen Bereich der fossilen Hohlform lagert ein eemzeitlicher Torf, dem altersmäßig der untere Boden entspricht (vgl. 4.2). Durch diese Datierung konnten die eingelagerten Sedimente altersmäßig abgeschätzt und genetisch klassifiziert sowie das Relief der Hohlform zu verschiedenen Zeiträumen, insbesondere auch während der Bodenbildungsphasen, rekonstruiert werden. Aufgabe dieser Arbeit ist es daher, einen Beitrag zur Reliefentwicklung seit der letzten Inlandvereisung am Ort, des Drenthe-2Vorstoßes, zu liefern.

Der Sandabbau wurde Ende 1980 eingestellt. Hauptsächlich wegen der fossilen Böden ist - mit Einverständnis des Besitzers, Ortsbürgermeister H. Peters - die verbliebene Abbauwand unter Naturschutz gestellt worden.

Die Untersuchungen in der Sandgrube Sonnen-Berg-NW geschahen im Rahmen eines von der Deutschen Forschungsgemeinschaft finanziell unterstützten Forschungsgrogrammes über weichselzeitliche Sedimente, ihre Genese und die entsprechende Reliefentwicklung in Nordwestdeutschland. Für diese Unterstützung sei der DFG herzlich gedankt. Ebenfalls danken möchten wir Herrn Prof. Dr. G. RoeschmanN, Niedersächsisches Landesamt für Bodenforschung in Hannover, für die ständige Beratung im Gelände und Herrn Prof. Dr. K.-E. BEHRE, Niedersächsisches Landesinstitut für Marschen- und Wurtenforschung in Wilhelmshaven, sowie Herrn Dr. E. Schulz, Geographisches Institut der Universität Würzburg, für pollenanalytische Untersuchungen.

\section{Arbeitsmethodik}

Der Aufschluß Sonnen-Berg-NW wurde etwa 2,5 Jahre ständig beobachtet und zusammen mit dem angrenzenden Gelände auf verschiedene Weisen untersucht.

Um die angeschnittenen Schichten flächenhaft verfolgen und das Altrelief rekonstruieren zu können, sind 25 Schlitzsondierungen niedergebracht worden. 16 davon wurden für Profilschnitte benutzt (vgl. Abb. 4). In den Grubenwänden wurden die Einregelungen von Geschiebelängsachsen und Schichteinfallen gemessen. Schließlich wurden folgende Laboruntersuchungen veranlaßt: Pollen- und Korngrößenanalysen sowie die Bestimmung von Zurundung und Glanz von Sandkörnern (vgl. Tab. 1).

Die Untersuchungsergebnisse dienten gleichzeitig als Grundlage für bodenkundliche Untersuchungen, die von Altemüller, Forschungsanstalt für Landwirtschaft in Braunschweig-Völkenrode, und Roeschmann durchgeführt worden sind. Darüber berichten beide gesondert.

\section{Lage, Geologie und Morphologie}

Etwa $1 \mathrm{~km}$ nordöstlich des Ortes Krempel (15 km S Cuxhaven) auf Bl. 2218 Wanna der TK 25 liegen zwei alte und überwiegend verfallene Sandgruben, von denen in der westlich gelegenen noch abgebaut wurde. Die Abbauwand befindet sich im Hangbereich nordwestlich des Sonnen-Berges, mit 26,8 m + NN höchster Punkt der näheren Umgebung (vgl. Abb. 1). Der Hang leitet zur breiten Niederung der Emmelke über. Diese Niederung ist Teil einer weit ins Binnenland, bis Bederkesa reichenden Bucht, die als Hadelner Bucht bezeichnet wird und nach DECHEND \& LANG (1965) ein drenthezeitliches Zungenbecken darstellt.

Der Sonnen-Berg samt seiner Umgebung liegt auf einer nach $\mathrm{E}$ auslaufenden und von der Altenwalder-Neuenwalder Geest vorspringenden Halbinsel. Stratigraphisch ist sie Teil der Altenwalder Staffel, eines N-S-streichenden Höhenzuges, der die äußerste Drenthe-2-zeitliche Eisrandlage im nördlichen Elbe-Weser-Dreieck darstellt (MEYER \&: SChNeEKLOTH 1973; LAde 1980). 


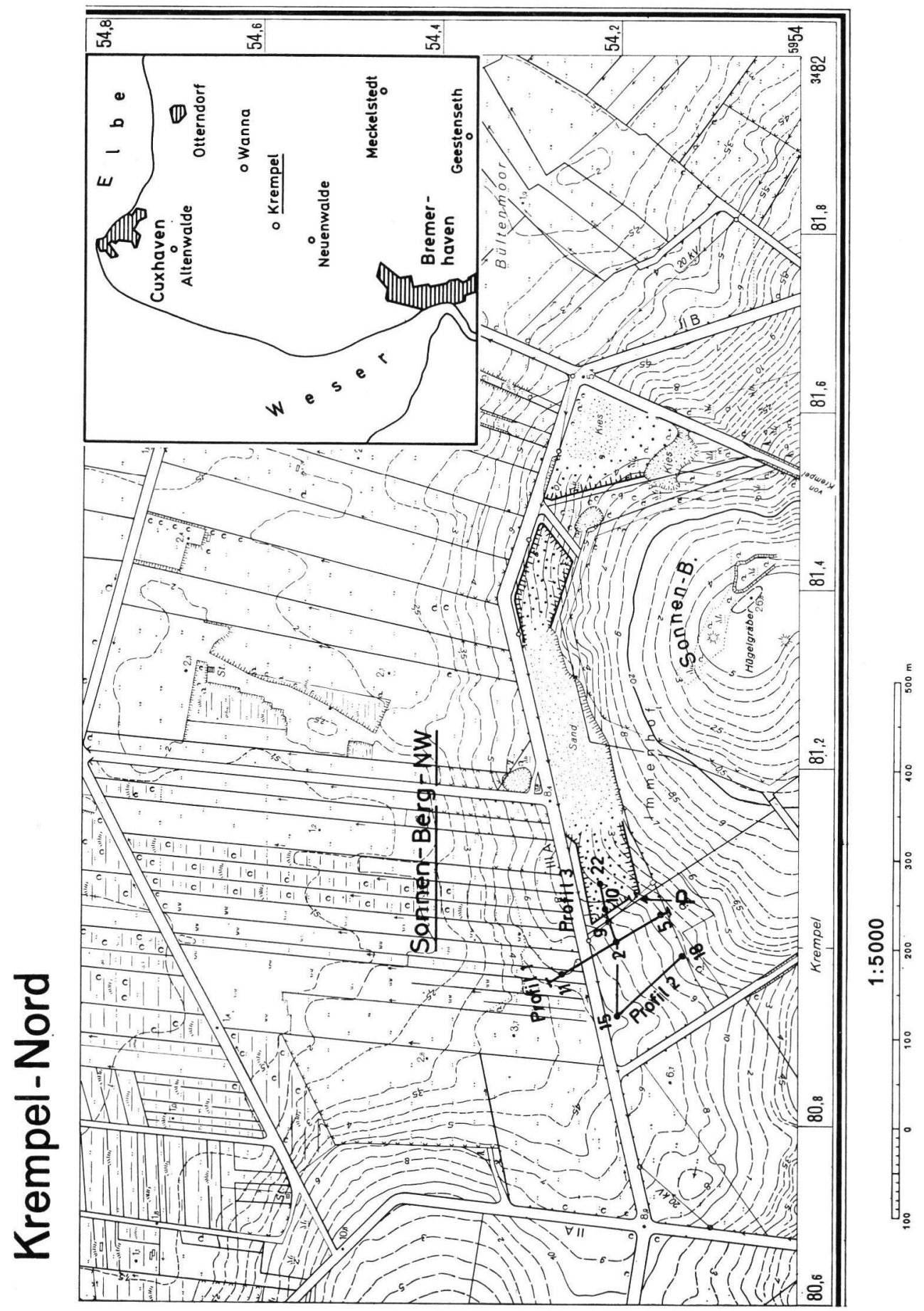

Abb. 1: Lage der Sandgrube Sonnen-Berg-NW, der Profile Sonnen-Berg-NW 1-3 und des Wandausschnittes P. - Verkleinerter Ausschnitt DGK 5.

Kartengrundlage: Deutsche Grundkarte 1:5000, 2218/26 Krempel-Nord, Ausgabe 1971.

Vervielfältigung mit Erlaubnis des Herausgebers: Katasteramt Wesermünde, Bremerhaven, den 18. 12. 1980 , A 2842/80. 
In der direkten Umgebung des Sonnen-Berges herrschen glazifluviatile Sande und Kiese in meist ungestörter Lagerung vor. Nur einmal wurde als eindeutig glaziäre Störung eine Falte beobachtet (direkt südlich Sondierung 22, fast auf Höhe der nächsten Feldgrenze; vgl. Abb. 1). Daneben wurden Geschiebelehm und glimmerhaltige Feinsande mit Schlufflagen angetroffen (vgl. Abb. 4). Andere Sondierungen, die nicht in den Profillinien liegen, stießen in grauen Geschiebemergel. All diese Schichten werden ins Drenthe-Stadium gestellt. Unsicher ist, ob sie Drenthe-1- oder Drenthe-2-zeitlich zur Ablagerung gekommen sind.

An anderen Hangbereichen der Geesthalbinsel Krempel tritt Geschiebemergel auf, der im oberen Teil viele Kreidegeschiebe führt und hellbraun und hellgrau gefärbt ist, im unteren Teil dagegen selten Kreidegeschiebe enthält, schwächer kalkig und grau gefärbt ist. Die Vorkommen liegen stets im Grundwasserbereich. Wegen der petrographischen Eigenheiten wird die Moräne dem Drenthe-2-Vorstoß, dem im Hamburger Raum der Niendorfer Vorstoß entspricht, zugeordnet. Danach könnte der graue Geschiebemergel am Sonnen-Berg ebenso alt sein.

An der Oberfläche der direkten Umgebung der Sandgrube Sonnen-Berg-NW, auch über der fossilen Hohlform, ist - wie fast überall in der Geest - eine Kies- und Steinbestreuung zu beobachten.

\section{Beschreibung}

\subsection{Sandgrube Sonnen-Berg-NW}

Anfang 1978 waren die Reste beider fossiler Böden noch in der Gänze aufgeschlossen und zeigten durch ihre konkave Lagerung eine Depression an, die nach den Sondierungen eine abflußlose Hohlform ist. Im Verlaufe des weiteren Abbaus wurde das Einfallen aller Schichten in Abbaurichtung beobachtet, wobei der untere Boden schneller als der obere abtauchte, d. h. die Mächtigkeit des zwischenlagernden Sandes zunahm. Bereits im Herbst 1978 lag im zentralen Bereich der Sandgrube der B-Horizont des unteren Bodens fast unter Grubensohle (vgl. Abb. 2). Bei Beendigung des Abbaus war die Oberkante des unteren Bodens in der Grubenmitte auf 2,5 m unter Grubensohle abgetaucht.

Nachfolgend werden die aufgeschlossenen Schichten und Horizonte (vgl. Abb. $2+3$ ) beschrieben. Die - zunächst bis zum Vorliegen weiterer bodenkundlicher Untersuchungsergebnisse vorläufige - bodengenetische Ansprache wurde freundlicherweise von RoEscHMANN vorgenommen.

\section{Schicht 1}

Weichselzeitliche, vermutlich weichselhochglaziale Fließerde mit holozänem Parabraunerde-Podsol (Oberboden abgeschoben).

Die Farbe des verbliebenen B-Horizontes variierte zwischen graubraunen und rotbraunen Tönen. Eine Korngrößenanalyse ergab (Gew.- $\%$ ) für Ton und Schluff ( $T+U)$ $23 \%$, Feinsand (fS) $29 \%$, Mittelsand (mS) $41 \%$, Grobsand (gS) $6 \%$ und Kies (G) $1 \%$. Gerölle und Steine mit Durchmessern bis zu mehreren Dezimetern sind stärker vertreten als die Analyse angibt. An der abgeschobenen Oberfläche tauchte stellenweise ein Stein-Pflaster auf, das vermutlich eine Anreicherung an der Oberkante eines ehemaligen Dauerfrostbodens ist. Wegen der gemischtkörnigen Zusammensetzung wird für die Gesamtschicht Bodenfließen, wahrscheinlich über Dauerfrostboden, angenommen. 


\section{S}

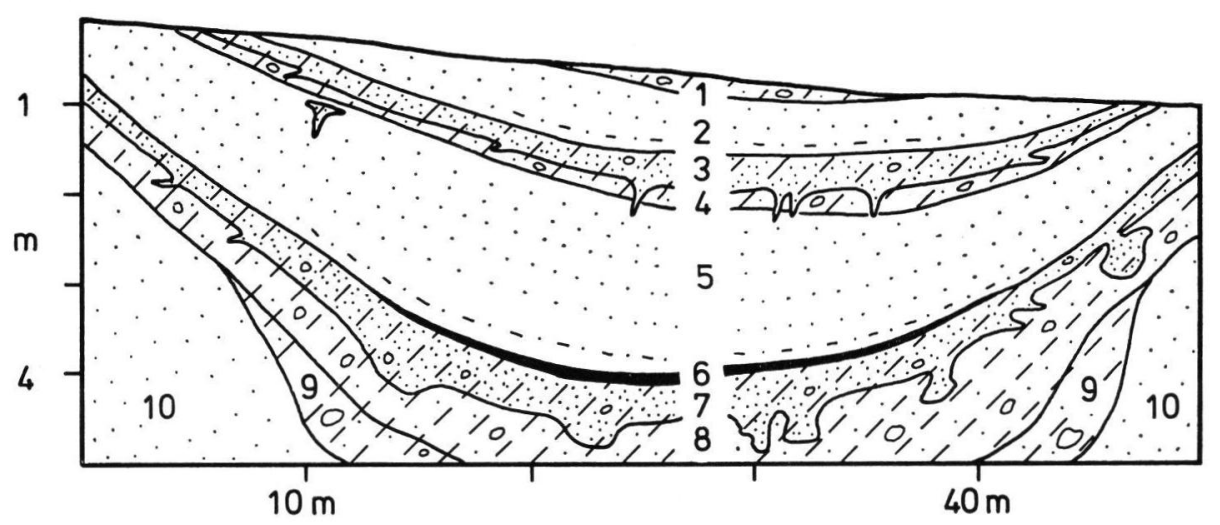

Abb. 2: Schematische Übersichtsskizze der Sandgrube Sonnen-Berg-NW auf Bl. 2218 Wanna (TK 25); Abbaustand: Herbst 1978.

\section{Schichten:}

1: Weichselzeitliche, vermutlich weichselhochglaziale Fließerde mit holozänem ParabraunerdePodsol (Oberboden abgeschoben).

2. Niveofluviatile Fein- und Mittelsande vermutlich des Brörup-Odderade-Stadials.

3: Eem-Brörup-stadiale Fließerde mit vermutlich brörupzeitlichem, periglaziär verändertem Bleichhorizont eines fossilen Bodens.

4: Eem-Brörup-stadiale Fließerde mit vermutlich brörupzeitlichem, z. T. periglaziär verändertem B-Horizont eines fossilen Bodens.

5: Eem-Brörup-stadiale niveofluviatile Fein- und Mittelsande.

6: Eem-Brörup-stadiale niveofluviatile Humusschicht.

7: Warthezeitliche Fließerde mit eemzeitlichem, periglaziär verändertem, z. T. umgelagertem Bleichhorizont eines fossilen Bodens.

8: Warthezeitliche Fließerde mit eemzeitlichem, periglaziär verändertem B-Horizont eines fossilen Bodens.

9: Warthezeitliche Fließerde.

10: Warthezeitliche niveofluviatile Sande.

\section{Schicht 2}

Niveofluviatile Fein- und Mittelsande vermutlich des Brörup-Odderade-Stadials mit rezenten $B_{t}-B a ̈ n d e r n$.

Der Sand war durchgehend hellbraun gefärbt und parallelgeschichtet. Im oberen Teil tauchten feine Gerölle, die z. T. lagenweise angeordnet waren, auf, während im unteren halben Meter sich zunehmend humose Lagen einschalteten, die als umgelagertes humoses Bodenmaterial des oberen fossilen Bodens gedeutet werden.

Stellenweise war die Schichtung durch Eiskeile und Frostspalten, die z. T. auch den darunter liegenden fossilen Boden durchstießen, gestört. Verwerfungen mit Versätzen im $\mathrm{dm}$-Bereich wurden ebenfalls beobachtet. Sie zeigen schichtweise Bewegungen im gefrorenen Zustand an, deren Ursache vermutlich Setzungen des in der Hohlform gebildeten Torfes und dadurch bedingte Schichtverschiebungen zum Hohlformtiefsten waren (vgl. Schicht 5). 


\section{Schicht 3}

Eem-Brörup-stadiale Fließerde mit vermutlich brörupzeitlichem, periglaziär verändertem

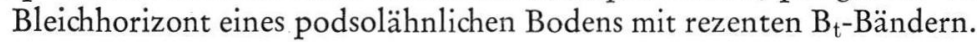

Der Horizont war im zentralen Bereich fast weiß, randlich weißgrau und durch isolierte kleine Pflanzenreste schwarz gesprenkelt sowie schichtungslos. Korngrößenmäßig handelt es sich um einen feinkiesigen und schwach schluffigen, z. T. auch stärker schluffigen Sand. In näherer Lage zur heutigen Oberfläche war stellenweise eine Kies-Sohle zu beobachten.

Aufpressungsformen, schräg bis zur obersten Fließerde verlaufende Verwerfungslinien und Humusschlieren unterschiedlicher Form belegen eine nachträgliche periglaziäre Beeinflussung. Auf kurzwegiges Bodenfließen sind hakenförmige Aufschleppungen an der Oberkante des B-Horizontes zurückzuführen. Wegen dieser Störungen ist der Bleichhorizont als parautochthon zu bezeichnen.

Die rezenten $B_{t}$-Bänder verliefen in der Regel schichtkonform und zeichneten Störungsstrukturen nach. Dies gilt auch für die hangenden Sande. Randlich bei größerer Nähe zur heutigen Oberfläche enthielten Bleichhorizont und hangende Sande bis fingerdicke und -lange Tierwühlgänge, die vermutlich erst im Holozän entstanden sind. Die Pflanzenreste waren durchweg schwarz gefärbt und bestanden - soweit an den Strukturen erkennbar - z. T. aus Stengeln und Blättern einer Bodenvegetation.

Wie bei Schicht 1 wird für die Genese dieses Substrates als auch das des liegenden BHorizontes Bodenfließen angenommen.

\section{Schicht 4}

Eem-Brörup-stadiale Fließerde mit vermutlich brörupzeitlichem, z. T. periglaziär verändertem B-Horizont eines podsolähnlichen Bodens.

Die Farbe dieses Horizontes variierte zwischen orangebraun und rostfarben; Schichtung war nicht zu erkennen. Nach einer Korngrößenanalyse, deren Probe aus dem zentralen Bereich der Grube stammt, verteilen sich die Fraktionen wie folgt: T: $9 \%$, U: $16 \%$, fS: $24 \%, \mathrm{mS}: 44 \%$, gS: $6 \%$ und G: $1 \%$. Steine bis Faustgröße waren vereinzelt enthalten. Da innerhalb der Schluff-Fraktion Grobschluff mit fast 9\% dominierte, ist ein erheblicher Feinkornanteil nicht pedogenetisch angereichert, sondern sedimentiert worden. D. h., daß bereits primär ein breites Korngrößenspektrum vorhanden war und daher sedimentgenetisch eine Fließerde angenommen werden kann.

Nachträgliche Störungen spiegelten sich eindrucksvoll in schräg stehenden bis waagerecht ausgezogenen, z. T. abgescherten (vgl. Abb. 3, Oberkante Schicht 5) Wurzelröhren wieder. Sie waren mit Bleichsand gefüllt und stießen z. T. bis in den liegenden Sand. Mehrfach wurden treppenförmige Strukturen mit Verlagerungsbeträgen bis $0,2 \mathrm{~m}$ beobachtet.

Zum Alter dieses Bodens und ergänzend zu den Ausführungen zu Schicht 2 sei erwähnt, daß aus der näheren und weiteren Umgebung mehrere fossile oder existierende Hohlformen mit organogenen Ablagerungen der Eem-Warmzeit und des Brörup-Interstadials bekannt geworden sind, so durch BeHre (1974) von Wanna und durch LADE (1980) von Meckelstedt und Geestenseth (vgl. Abb. 1). Die eingelagerten Schichten dort sind denen hier vergleichbar.

Bei eigenen Untersuchungen in einer anderen Sandgrube, die etwa 1,3 km NNW Sandgrube Sonnenberg-Berg-NW in derselben morphologischen Position liegt und ebenfalls zwei fossile Böden beherbergt (aus einer weiteren Grube $300 \mathrm{~m}$ weiter westlich beschreibt HeNRICI bereits 1962 zwei fossile Böden), wurden in $100 \mathrm{~m}$ Entfernung in Richtung Niederung zwei verschiedene Torfe erfaßt, die von Behre (schr. Mitt. v. 15. 1. 79) in die Eem-Warmzeit und in die erste Hälfte des Brörup-Interstadials datiert wurden. Leider ist der Versuch, mit Hilfe von Sondierungen Torfe und Böden lückenlos miteinander zu verbinden, nicht voll geglückt. Stellenweise fehlte ein Boden, 


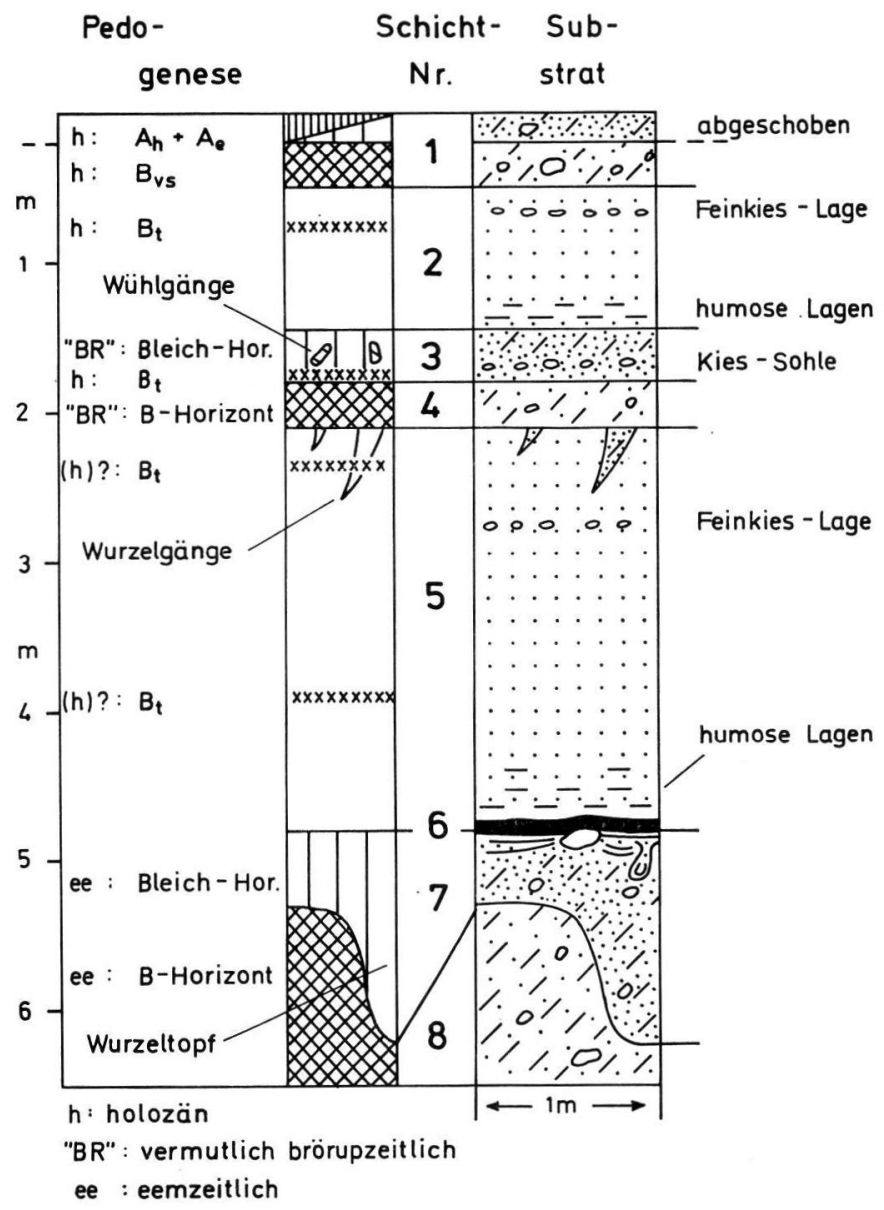

Abb. 3: Wandausschnitt P in Sandgrube Sonnen-Berg-NW auf Bl. 2218 Wanna (TK 25); Abbaustand: Herbst 1980; Lage: vgl. Abb. 1.

\section{Substrat:}

1: tonig-schluffig-kiesig-steiniger Sand

2: Fein- und Mittelsande

3: schluffig-feinkiesiger Sand

4: tonig-schluffig-kiesiger Sand mit einzelnen Steinen

5: Fein- und Mittelsande

6: Humusschicht, z. T. mit mineralischem Anteil

7: schluffig-kiesiger Sand mit sehr geringen Ton- und geringen Steinanteilen

8: tonig-kiesig-steiniger Sand mit hohen Schluffanteilen.

was z. B. auf farblich schwache Ausbildung, nachträgliche Störungen oder auch Erosion zurückgeführt werden kann. Trotzdem wird angenommen, daß die beiden Böden den beiden Torfen zeitlich entsprechen.

Diese Beispiele unterstützen die Einstufung in das Brörup-Interstadial. 


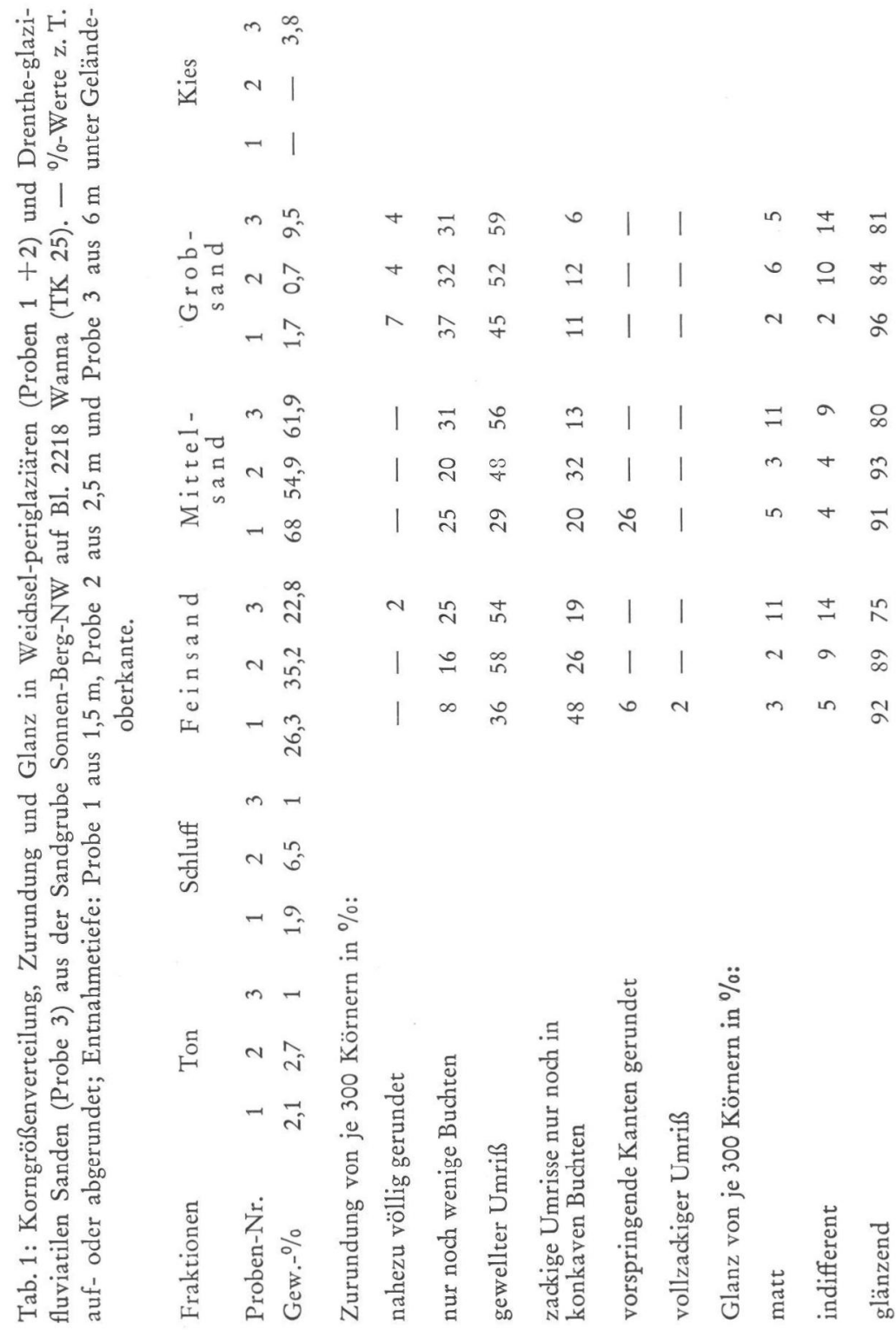

Schicht 5

Eem-Brörup-stadiale niveofluviatile Fein- und Mittelsande mit rezenten (?) $\mathrm{B}_{\mathrm{t}}$-Bändern.

Der Sand war gelblich-hellbraun und im unteren Bereich, wo z. T. Bleichsand umgelagert worden ist, weiß gefärbt sowie stets parallelgeschichtet. Die Korngrößenverteilung des gelblich-hellbraunen Sandes aus etwa der Mitte dieser Schicht geht aus Tab. 1 hervor. Zum Hangenden und Liegenden war Feinkies, teilweise zu Lagen angeordnet, beigemengt. Humose Lagen waren im unteren Teil auch zu beobachten. Die Anzahl der rezenten (?) $\mathrm{B}_{\mathrm{t}}$-Bänder war deutlich geringer als in den oberen Sanden. 
Außer den erwähnten Schichtstörungen, die vorwiegend im Randbereich der Hohlform in diese Schicht reichten, wurden im zentralen Bereich zweimal Aufschiebungen, in die humose Lagen einbezogen waren, beobachtet. An anderer Stelle war ein Eiskeil, der sich bis in den eemzeitlichen B-Horizont fortsetzte, durch zwei nacheinander ablaufende Rutschungen in eine Treppe mit zwei Stufen zerhackt. Diese Form ist wohl so zu erklären, daß nach der ersten Bewegung, die den unteren Teil der Spalte hat stehen lassen, ein oberer Teil der vorher bewegten Schicht noch einmal rutschte. Insgesamt machte der horizontale Versatz etwa $0,5 \mathrm{~m}$ aus.

In Schalkholz sind solche Sande von Menke (1976) genetisch als überwiegend niveofluviatil mit geringer äolischer Komponente angesprochen worden. Um diese Frage für Sonnen-Berg-NW zu klären, wurden zwei Proben aus dieser Schicht auf Zurundung und Glanz untersucht. Aus Vergleichsgründen wurde eine dritte, die aus den glaziär gefalteten Sanden derselben Grube stammt (vgl. 3.), hinzugenommen.

Das Ergebnis ist in Tab. 1 dargestellt. Danach ist die Zurundung gut, Mattierung selten. Entscheidend für die genetische Einstufung ist, daß dieser Befund auch für die glazifluviatilen Sande gilt. In der Grobsand- und Mittelsand-Fraktion ist die Zurundung sogar besser, während der Anteil von mattierten Körnern in der Feinsand- und Mittelsand-Fraktion höher ist. Daraus wird geschlossen, daß die weichselstadialen Sande keine Flugsande, sondern überwiegend niveofluviatile Sande sind und als Lieferschicht nur die hangaufwärts unter einer periglaziären Deckschicht anstehenden glazifluviatilen Sedimente des Drenthe-Stadiums in Frage kommen (zur Herkunft vgl. auch 4.3.).

\section{Schicht 6}

Eem-Brörup-stadiale niveofluviatile Humusschicht.

Die Farbe dieser Schicht war überwiegend schwarz, lagenweise auch schwarzgrau oder graubraun. Durch die Farbdifferenzierung wird ein unterschiedlicher Mineralgehalt und gleichzeitig eine Schichtung angezeigt. Gedeutet wird diese Schicht als selektiv umgelagertes humoses Bodenmaterial des liegenden Eem-Bodens. Sie war nicht stärker als $0,05 \mathrm{~m}$ und beschränkte sich stets auf den jeweils tiefsten Teil der angeschnittenen Hohlform (vgl. Abb. 2). Strukturen nachträglicher Störungen kamen nur in Form von Aufspaltungen vor. Die Auflagerung des hangenden Sandes muß daher, wie in Schalkholz (Menke 1976), sehr schonend abgelaufen sein. Im Gegensatz zu Schalkholz stellt diese Schicht ein Sediment, keinen Horizont dar.

\section{Schicht 7}

Warthezeitliche Fließerde mit eemzeitlichem, periglaziär verändertem, z. T. umgelagertem Bleichhorizont eines Eem-Bodens mit Parabraunerde- und Podsol-Merkmalen.

Im Gegensatz zum Bleichhorizont des oberen fossilen Bodens war dieser durchgehend weiß. Das Substrat zeigte sich als schwach steiniger, kiesiger, sehr schwach tonig-schluffiger, im unteren Teil stark schluffiger Sand. Ton- und Schluffgehalt nahmen zum Rand hin generell ab. Rezente (?) $\mathrm{B}_{\mathrm{t}}$-Bänder kamen bis in diesen Horizont vor.

Neben Wurzelröhren erschienen sackförmige Wurzeltöpfe, die sich teilweise bis über $1 \mathrm{~m}$ tief ins Liegende stülpten und ebenfalls mit Bleichsand gefüllt waren.

Für die beobachteten Schichtstörungen waren hauptsächlich solifluidale Bewegungen und Umlagerungen, die im oberen Teil dieses Horizontes z. T. Fließschichtungsstrukturen hinterlassen haben, verantwortlich. Daneben erschienen wurzellose Schollen und fahnenartig ausgezogene Streifen von B-Horizont-Material. Kryogene Strukturen schienen die 
unterschiedlich geformten Humusschlieren $\mathrm{zu}$ sein. In Wandausschnitt P bildeten zwei davon einen „Tropfen“ (vgl. Abb. 3). Es entstand der Eindruck, als wären die benachbarten Humusschlieren „Tropfen“ im Initialstadium.

Die sedimentgenetische Einstufung als Fließerde geschah nach den gleichen Gesichtspunkten wie für die oberen Fließerden auch. Da der Bleichhorizont trotz aller Umlagerungen und Störungen seinen Charakter behalten hat und, bezogen auf A- und B-Horizont, die Horizontierung erhalten geblieben ist, soll er hier noch als parautochthon bezeichnet werden.

\section{Schicht 8}

Warthezeitliche Fließerde mit eemzeitlichem, periglaziär verändertem B-Horizont eines Eem-Bodens mit Parabraunerde- und Podsol-Merkmalen.

Je nach morphologischer Position und Ausgangssubstrat - zentral etwas ton- und schluffreichere, randlich sandige Fließerde - besaß der B-Horizont makromorphologisch Merkmale eines $\mathrm{B}_{\mathrm{t}^{-}}$und/oder eines $\mathrm{B}_{\mathrm{sh}}$-Horizontes. Entsprechend waren die Farben in den tieferen Lagen der Hohlform graubraun bis rotbraun gegenüber leuchtend orangerot in den höheren Randbereichen. Eine Korngrößenanalyse aus dem zentralen Bereich der Sandgrube ergab: T: 4,5\%, U: $28 \%$, fS: $35 \%$, mS: $25 \%$, gS: $5 \%$ und Kies: 2,5\%, wobei Geschiebe bis zu mehreren dm $\phi$ ebenfalls enthalten waren. Da allein Grobschluff mit $18 \%$ beteiligt war, dürfte auch hier der Feinkornanteil größtenteils sedimentiert worden sein.

An Störungen sind hauptsächlich hangabwärts verbogene Wurzelgänge und -töpfe zu nennen. Deshalb können die unteren Teile der B-Horizonte als autochthon, die oberen als parautochthon angesprochen werden, während sedimentgenetisch eine Fließerde vorliegt.

\section{Schicht 9}

\section{Warthezeitliche Fließerde.}

Die Schicht war durchgehend grüngrau gefärbt und von der Korngrößenzusammensetzung dem hangenden B-Horizont in tieferen Lagen der Hohlform vergleichbar.

An Schichtstörungen wurden Sackungsstrukturen mit Sprunghöhen im Meterbereich beobachtet. Ursache war vermutlich abtauendes drenthezeitliches Toteis (vgl. 4.2.). Da nach Menke (1976: 66) Toteis wenigstens bis in die frühe Eichen-Mischwald-Zeit (Eem IV a, b) überdauern konnte, ist hier mit Sackungen sowohl im Warthe-Stadium als auch in der beginnenden Eem-Warmzeit zu rechnen. D. h., diese Schicht sowie die Substrate der hangenden eemzeitlichen Bodenhorizonte haben vermutlich eine Warthe-periglaziäre Vorprägung erfahren und sind Weichsel-periglaziär noch einmal überformt worden.

Um Hinweise auf die Genese dieser Schicht - Grundmoräne in situ oder Fließerde zu bekommen, wurden in einem schmalen Ausschnitt die Einregelungen von 50 Geschiebelängsachsen gemessen. Das Diagramm war jedoch nicht voll aussagekräftig und zeigte Werte zwischen $240^{\circ}$ und $360^{\circ}$ mit Spitzen bei $270^{\circ}, 290^{\circ}$ und $325^{\circ}$. Vom Meßbereich aus wies keine Richtung zum Hohlformzentrum. Die stärkste Wertehäufung ergab sich bei $290^{\circ}$, was genau der lokalen Hangrichtung entsprach. Dies könnte ein Hinweis darauf sein, daß eine Fließerde vorliegt, die vor Hohlformentstehung, etwa im Warthe-Hochglazial, hangabwärts geflossen ist. Es soll aber nicht verschwiegen werden, daß in Ablationsmoränen ähnliche Einregelungsdiagramme erzielt werden. 


\section{Schicht 10}

Warthezeitliche niveofluviatile Sande.

Die Sande waren gelblich-hellbraun gefärbt, undeutlich geschichtet und mit einer schwach rostfarbenen, ebenfalls undeutlichen Bänderung versehen. Möglicherweise handelt es sich z. T. um fossile $B_{t}$-Bänder. Während das Sediment im nördlichen Grubenbereich als Feinsand mit Schlufflagen ausgebildet war, zeigte es sich im südlichen Grubenbereich als Mittel- und Feinsand mit Grobsandlagen. Hier waren diese in Anlehnung an die oben erwähnte Falte sedimentiert und nicht in die Stauchung einbezogen. Aus diesem Grunde werden sie als niveofluviatile Sande gedeutet.

\subsection{Profile Sonnen-Berg-NW}

Einige der in Abb. 4 dargestellten Sondierungen haben anstelle des unteren fossilen Bodenrestes einen Torf angetroffen, der in einer Hohlform gewachsen ist. Diese ist eingetieft in warthezeitliche niveofluviatile Sande mit Fließerdelagen. Die größte Mächtigkeit dieser Schicht wurde in Sondierung 1 mit fast $8 \mathrm{~m}$ festgestellt. Leider sind die Verhältnisse im Zentrum der Hohlform nicht vollständig bekannt, da die Sondierungen 2, 3 und 9 wegen zu großen Reibungswiderstandes aufgegeben werden mußten. Unter den Warthe-periglaziären Sedimenten folgten meist Schmelzwassersande und -kiese, daneben Geschiebelehm und mehrfach Beckenfeinsande, die alle dem Drenthe-Stadium zugeordnet werden.

Das Zentrum der Hohlform liegt etwa bei Sondierung 9. Eine Bestätigung dessen ergab auch eine Messung in den Eem-Brörup-stadialen Sanden der Schicht 5 (vgl. Abb. 2) etwa $10 \mathrm{~m}$ nördlich Sondierung 10. Dort fielen sie mit $35^{\circ}$ genau in Richtung Sondierung 9 ein. Der Torf besaß im Zentrum eine Mächtigkeit von 3,7 m und war stark gepreßt, so daß Sondierung 9 bei $12 \mathrm{~m}$ unter Gelände $(0,6 \mathrm{~m}$ unter Torfbasis) aufgegeben wurde. Petrographisch bestand der obere Meter aus stark zersetztem Hochmoortorf mit Wollgrasresten und die restliche Strecke aus mäßig bis stark zersetztem Bruchwaldtorf mit vielen Erlenholzresten. 2 Proben wurden von BEHRE pollenanalytisch untersucht und in die Eem-Warmzeit datiert: bei 11,3 m Hainbuchen-Zeit (Eem V) und bei 8,6 m KiefernFichten-Tannen-Zeit (Eem VI; schr. Mitt. v. 15. 1. 79). Weitere Proben aus den Sondierungen 1 und 2 wurden von Schulz untersucht und alle in die Kiefern-Zeit (Eem VII) datiert.

Der Nachweis, daß unterer Boden und benachbarter Torf in derselben Wärmephase entstanden sind, konnte über die Abgrenzung durch die unter- und überlagernden Schichten geschehen. Ein Vergleich der Sondierungen 1, 10 und 22 in Kombination mit dem Aufschluß ergab, daß das Liegende nach Farbe, Korngrößenzusammensetzung und Schichtenfolge jeweils gleich oder sehr ähnlich ausgebildet war. Nach oben folgten stets dieselben Fein- und Mittelsande und - außer in Sondierung 1 - der obere fossile Boden. Als beste Leitschicht erwies sich jedoch die Humusschicht (Schicht 6), die in der Sandgrube zusammen mit dem oberen Teil des Bleichhorizontes das älteste weichselzeitliche Sediment darstellt. Die Humusschicht lag in Sondierung 10 direkt dem Torf auf. Durch diese Eingrenzung von Torf und Boden ist die mittelbare Datierung des unteren fossilen Bodens in die Eem-Warmzeit gesichert.

Es bleibt die Erörterung der Hohlformgenese. Insbesondere aus Profil 1 ist ablesbar, daß sich die Warthe-periglaziären Sedimente in die liegenden glazifluviatilen Sande eingestülpt haben. Hierdurch wird eine Sackung angezeigt, deren Ursache vor Ablagerung der warthezeitlichen Schichten angelegt gewesen sein muß. Am ehesten kann dafür verschüttetes drenthezeitliches Toteis verantwortlich gemacht werden. 

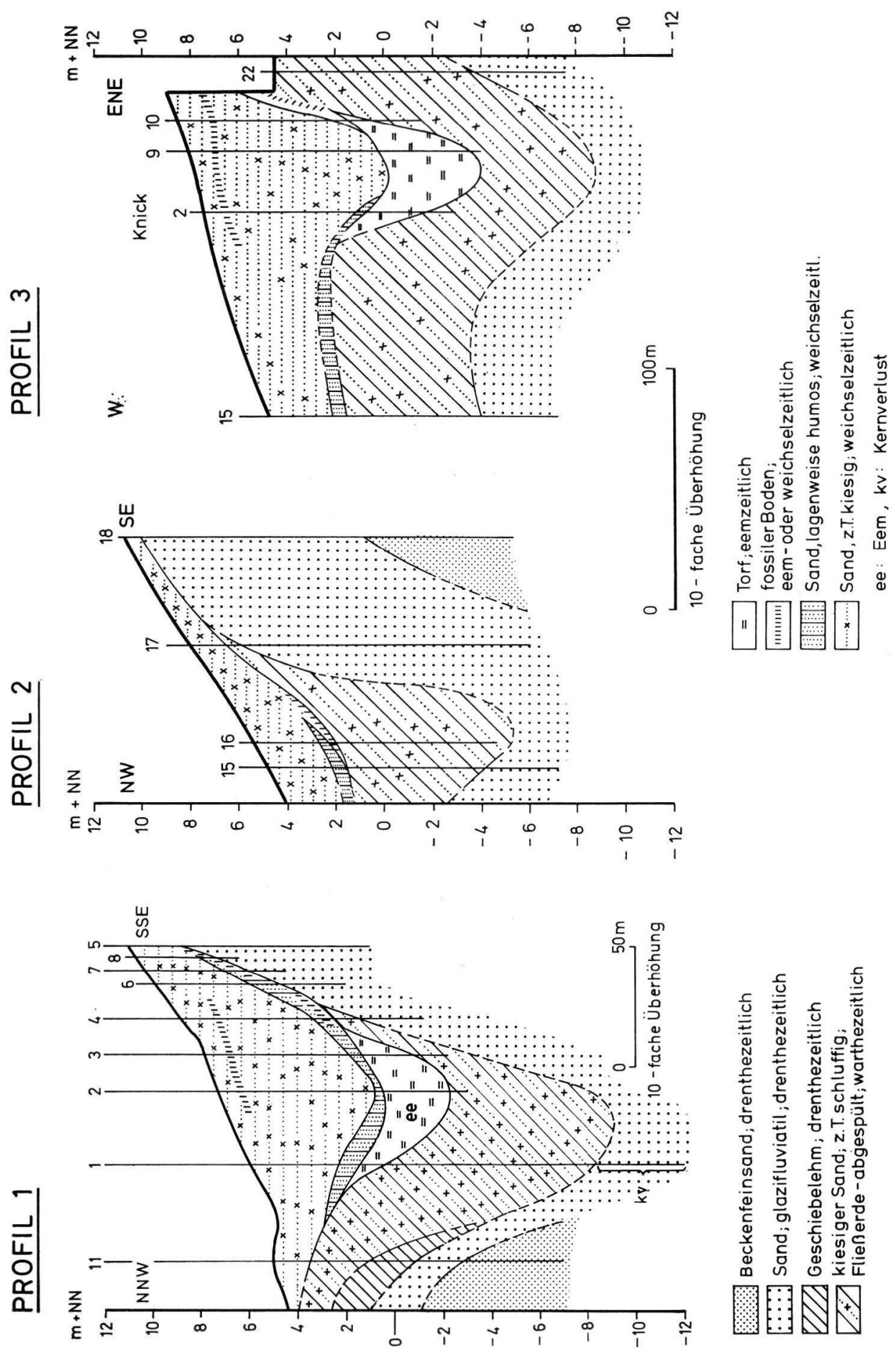

Abb. 4: Profile Sonnen-Berg-NW 1-3; Lage: vgl. Abb. 1. 


\subsection{Reliefentwicklung}

Nach den vorangegangenen Ausführungen hat sich seit Inaktivierung des Drenthe-2Eises am Ort das lokale Relief wie folgt entwickelt:

Im Bereich der eemzeitlichen Hohlform und benachbart sedimentierten sich warthezeitlich hauptsächlich niveofluviatile Sande und - untergeordnet - geringmächtige Fließerden. Anschließend, wahrscheinlich unter Klimaverschlechterung im Warthe-Hochglazial, wurde eine schluffig-sandige Fließerde aus Grundmoränenmaterial abgelagert, die noch kryoturbat überformt wurde.

Vermutlich schon vor Beginn der Eem-Warmzeit setzten infolge ersten Abschmelzens von verschüttetem Toteis Sackungen ein, die ein verstärktes Sedimentieren im Hohlformbereich zur Folge hatten. Darauf weist die Mächtigkeitszunahme der entsprechenden Schichten in den Sondierungen 1, 15 und 16 hin. Der restliche Teil des Toteises schmolz in den ersten Phasen der Eem-Warmzeit ab und verursachte eine etwa $6 \mathrm{~m}$ tiefe Hohlform.

Zunächst bildete sich dort ein Bruchwald- und in der Kiefern-Zeit ein Hochmoortorf, der die Oberfläche der lokalen Umgebung überragte. Diese Reliefverhältnisse ergeben sich nicht nur aus allgemeinen Erkenntnissen über das Wachstum von Hochmooren, sondern auch aus einer Abschätzung des Setzungsausmaßes. Über Vergleiche der Oberkanten des Bruchwaldtorfes berechnet sich dessen ehemalige Oberfläche auf etwa $2 \mathrm{~m}+\mathrm{NN}$, was einen Sackungsquotienten von etwa $50 \%$ bedeutet. Ubertragen auf den Torf in Sondierung 9 errechnet sich die ehemalige Oberfläche dort bei etwa $4 \mathrm{~m}+\mathrm{NN}$. Das bedeutet, daß am Ende der Kiefern-Zeit die Hohlform - möglicherweise bis auf einen gewissen randlichen Bereich - verfüllt war und das aufgesetzte Hochmoor die mineralische Umgebung überragte. Dies ist wahrscheinlich der Grund, warum im ersten Weichselstadial der zentrale Bereich der vermoorten Hohlform von der Sedimentation der Humusschicht (Schicht 6) und der aufliegenden Sande mit Humuslagen (unterer Teil Schicht 5) ausgespart wurde.

$\mathrm{Zu}$ Beginn des Eem-Brörup-Stadials setzten im trockenen Randbereich der Hohlform Umlagerungen in Richtung Torf ein, die zunächst den eemzeitlichen humusreicheren Oberboden und anschließend den Bleichhorizont betrafen. Entsprechend dieser Reihenfolge liegt im Sedimentationsgebiet die Humusschicht unter dem Bleichsand-Sediment. Gleichzeitig kam es am Hang zu kurzwegigen Verlagerungen des nicht abgetragenen Bodenrestes durch Solifluktion.

Im weiteren Verlauf dieses Stadials wurden durch das an der Oberfläche ablaufende Wasser Sande in die Hohlform transportiert, die sich schließlich auch dem Torf auflagerten und seine Setzung bzw. Sackungen an der Oberfläche verursachten. Die Hohlform entstand im Bereich des Torfes neu. Da der Flugsandanteil in den Sanden sehr gering ist, kommen als Lieferschichten nur Drenthe-2-Grundmoräne und glazifluviatile Sedimente in Frage. Geschiebelehm oder -mergel können als Hauptlieferanten ausgeschlossen werden, da sonst neben Sand auch die feineren Fraktionen Ton und Schluff abgespült worden wären und an der Hohlformfüllung hätten beteiligt sein müssen (nach Höfle \& SCHLENKER [1979] haben z. B. in der frischen Drenthe-2-Moräne in der Kreidegrube Hemmoor Ton und Schluff einen Anteil um $40 \%$ ).

Die Umlagerung der glazifluviatilen Sedimente in den Hangbereichen oberhalb der Hohlform muß überwiegend linienhaft erfolgt sein, da das Anstehende dort flächenhaft von Drenthe-2-Grundmoräne oder ihrem Umlagerungsprodukt Fließerde gebildet wurde (wie heute auch). Das Vorhandensein jüngerer Fließerden in der Hohlform bestätigt dies. Danach haben die Schneeschmelzwässer Rinnen bis in die glazifluviatilen Schichten eingetieft und in diesen Bahnen den Sand transportiert. 
Gegen Ende des Eem-Brörup-Stadials änderte sich der Sedimentationsmechanismus, und es lagerte sich eine Fließerde ab. Die Vergröberung der liegenden niveofluviatilen Schicht nach oben weist auf feuchtere Bedingungen und damit auf Klimaverschlechterung hin. Nach Beendigung der Sedimentation in diesem Stadial kann nur noch eine sehr flache Hohlform existiert haben, wie die Lagerung des oberen fossilen Bodens in den Profilen 1 und 3 (Abb. 4) und in der Sandgrube (Abb. 2) zeigt. Denkbar ist auch, daß sie derzeit verfüllt war und durch die beobachteten Sackungen (vgl. Schichten 3 u. 4) kurzzeitig und als flache Schale neu entstand.

Auf der Fließerde und stellenweise unter Einbeziehung der liegenden Sande bildete sich vermutlich im Brörup-Interstadial im Hohlformbereich ein Boden mit Podsol-Merkmalen. Die Mächtigkeit seiner Horizonte sind in vergleichbarer Reliefposition geringer als die des Eem-Bodens. Allerdings kann bei Beobachtung nur eines fossilen Bodens aus den Mächtigkeiten der Horizonte und vielleicht der Intensität der Horizontfarben allein in der Regel nicht auf bestimmte klimatische Bildungsbedingungen (z. B. Interglazial oder Interstadial) sowie auf ein bestimmtes Alter geschlossen werden. Solange keine mittelbare oder unmittelbare Datierung vorliegt, sind Kenntnisse der Lage des fossilen Bodens im Altrelief unbedingt notwendig (RoEschmann 1975).

Im Anschluß an die Bildung des oberen fossilen Bodens, vermutlich im Brörup-Odderade-Stadial, liefen Abtragungs- und Ablagerungsvorgänge $a b$, die denen im vorangegangenen Stadial sehr ähnlich sind und deshalb nicht wiederholt werden sollen. Spätestens mit Abschluß der Sandsedimentation in diesem Stadial war die Hohlform verfüllt. $\mathrm{Zu}$ dem Zeitpunkt hatte sich im Hohlformbereich ein Gleichgewicht zwischen Abtragung und Ablagerung eingestellt. Jüngere Böden aus den Interstadialen Odderade und „Keller“ (MENKE 1976) und zwischenlagernde periglaziäre Schichten wurden nicht beobachtet. Die oberste Fließerde, auf der sich der holozäne Boden gebildet hat, ist vermutlich erst im Hochglazial der Weichsel-Kaltzeit abgelagert worden.

Nach der geschilderten Reliefentwicklung lassen sich mehrere Zyklen hinsichtlich Klima, Transport- bzw. Bildungsvorgang und Sediment bzw. Bildungsprodukt ableiten. So begann z. B. das erste Weichselstadial mit der Sedimentation von niveofluviatilen Schichten und endete unter Klimaverschlechterung mit der Sedimentation einer Fließerde. In einer anschließenden wärmeren Phase, vermutlich im Brörup-Interstadial, bildete sich ein Boden. Ein ähnlicher Zyklus kann für Warthe-Stadium und Eem-Warmzeit ebenfalls aufgestellt werden, während der jüngste Zyklus durch Schichten nicht vollständig repräsentiert ist. Wenn der Zeitraum in einem Weichselstadial mit Klimaverschlechterung, entsprechend dem Terminus Hochglazial, mit Hochstadial bezeichnet wird, ergibt sich verallgemeinert der folgende schematische Zyklus:

Stadial

Hochstadial/-glazial

Interstadial/-glazial

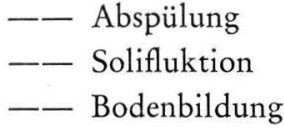

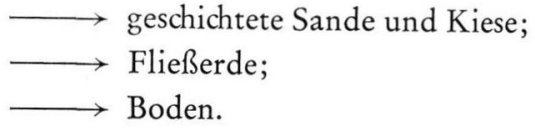

\section{Schriftenverzeichnis}

BeHre, K.-E. (1974): Die Vegetation im Spätpleistozän von Osterwanna/Niedersachsen. - Geol. Jb., A 18: 3-48, 8 Abb., 5 Taf.; Hannover.

Daнms, E. (1972): Limnogeologische Untersuchungen im Dümmer-Becken im Hinblick auf seine Bedeutung als Natur- und Landschaftsschutzgebiet. - Diss. FU Berlin, 231 S., 56 Abb., 3 Tab., 2 Taf.; Berlin.

Dechend, W. \& Lang, H. D. (1965): Die geologische Entwicklung der Hadeler Marsch. - Jb. Männer vom Morgenstern, 46: 9-23, 4 Abb.; Bremerhaven. 
DüCKER, A. (1967): Interstadiale Bodenbildungen als stratigraphische Zeitmarken im Ablauf der Weichsel-Kaltzeit in Schleswig-Holstein. - Fundamenta, B/2: 30-73; Köln-Graz.

- \& Hummel, P. (1967): Die fossilen Böden von Odderade/Dithmarschen, ein Beitrag zur Stratigraphie der Weichsel-Kaltzeit. - Fundamenta, B/2: 80-100; Köln-Graz.

Ehlers, J. (1978): Die quartäre Morphogenese der Harburger Berge und ihrer Umgebung. Mitt. Geogr. Ges. Hamburg, 68: 181 S., 62 Abb., 28 Taf., 1 Kt.; Hamburg.

Felix-Henningsen, P. (1979): Merkmale, Genese und Stratigraphie fossiler und reliktischer Bodenbildungen in saalezeitlichen Geschiebelehmen Schleswig-Holsteins und Süd-Dänemarks. Diss. Univ. Kiel: 219 S., 45 Abb., 11 Tab., 1 Beil.; Kiel.

GARLEFF, K. (1968): Geomorphologische Untersuchungen an geschlossenen Hohlformen (Kaven“) des Niedersächsischen Tieflandes. - Gött. Geogr. Abh., 44: 142 S., 13 Abb., 1 Kt.; Göttingen.

Grahle, H.-O. \& MüLleR, H. (1967): Das Zwischenahner Meer. - Oldenburger Jb., 66: 83-121, 13 Abb., 1 Taf.; Oldenburg.

Grube, F. (1967): Die Gliederung der Saale-(Riß-)Kaltzeit im Hamburger Raum. - Fundamenta, B/2: 168-195, Tab. 28, Taf. 37-44; Köln-Graz.

HENRICI, H. (1962): Kartierbericht zur Bodenkundlich-geologischen Karte der Marschengebiete, Bl. Wanna (2218). - Manuskript, Archiv Niedersächsisches Landesamt für Bodenforschung; Hannover. - [Unveröff.]

HöFle, H.-C. \& Schlenker, B. (1979): Das Pleistozänprofil der Kreidegrube Hemmoor bei Stade (Elbe-Weser-Dreieck). - Geol. Jb., A 49: 3-25, 10 Abb., 2 Tab.; Hannover.

LADE, U. (1974): Der Wollingster See - ein Beitrag zum Alter und zur Entstehung. - Jb. Männer vom Morgenstern, 54: 9-32, 6 Abb., 1 Taf.; Bremerhaven.

- (1979): Neuere Untersuchungen am Wollingster See. - Jb. Männer vom Morgenstern, 58: 11-26, 2 Abb.; Bremerhaven.

- (1980): Quartärmorphologische und -geologische Untersuchungen in der Bremervörder-Wesermünder Geest. - Würzburger Geogr. Arb., 50: 176 S., 39 Abb., 3 Taf., 2 Kt.; Würzburg.

MenKe, B. (1976): Neue Ergebnisse zur Stratigraphie und Landschaftsentwicklung im Jungpleistozän Westholsteins. - Eiszeitalter u. Gegenwart, 27: 53-68, 1 Abb., 3 Tab.; O' Ohringen.

Meyer, K.-D. (1973): Zur Entstehung der abflußlosen Hohlformen auf der Neuenwalder Geest. - Jb. Männer vom Morgenstern, 53: 23-29, 1 Abb., 1 Taf.; Bremerhaven.

- \& Schneekloth, H. (1973): Erläuterungen zu Blatt Neuenwalde Nr. 2318. - Geol. Kt. Niedersachsen 1 : 25 000: 80 S., 12 Abb., 1 Tab., 3 Taf., 1 Kt.; Hannover.

PiCARD, K. (1959): Gliederung pleistozäner Ablagerungen mit fossilen Böden bei Husum/Nordsee. - N. Jb. Geol. Paläont. Mh., 6: 259-272, 4 Abb.; Stuttgart.

- (1961): Reste von Pingos bei Husum/Nordsee. - Schr. Naturw. Ver. Schl.-Holstein., 32: 72-77, 2 Abb.; Kiel.

- (1964): Abflußlose Senken in Schleswig-Holstein. - Die Heimat, 71: 147-150; Neumünster.

Roeschmann, G. (1975): Zur Untersuchungsmethodik, pedogenetischen Deutung und Datierung fossiler Sandböden des Pleistozäns in Norddeutschland. - Mitt. Deutsch. Bodenkdl. Ges., 22: 581-590, 1 Abb.; Göttingen.

Semmel, A. (1969): Verwitterungs- und Abtragungserscheinungen in rezenten Periglazialgebieten (Lappland und Spitzbergen). - Würzburger Geogr. Arb., 26: 95 S., 15 Abb., 2 Tab., 13 Taf.; Würzburg.

Sindowski, K.-H. (1965): Die drenthestadiale Altenwalder Stauchmoräne südlich Cuxhaven. Z. dt. geol. Ges., 115: 158-162, 3 Abb.; Hannover.

Stremme, H. E. (1964): Die Warmzeiten vor und nach der Warthe-Eiszeit in ihren Bodenbildungen bei Böxlund (westl. Flensburg). - N. Jb. Geol. Paläont. Mh., 4: 237-247, 3 Abb., 2 Tab.; Stuttgart.

Geol. Landesamt Schleswig-Holstein (1980): Quartär-Exkursionen in Schleswig-Holstein (bearb. v. Stremme, H. E. \& Menke, B.): 132 S., 39 Abb., 5 Tab., 4 Beil.; Kiel. 
